\title{
Transmission Strategies for Throughput Maximization in High Speed Train Communications: From Theoretical Study to Practical Algorithms
}

\author{
Ayotunde O. Laiyemo, Student Member, IEEE, Harri Pennanen, Member, IEEE, \\ Pekka Pirinen, Senior Member, IEEE, and Matti Latva-aho, Senior Member, IEEE
}

\begin{abstract}
This paper focuses on improving the downlink throughput of the base station to train communication link in a high speed train (HST) scenario. First, we provide a theoretical study of the throughput maximization problem in a singlecell MIMO-OFDM train scenario with and without cooperation among carriages. The aim is to give fundamental insight into the problem rather than providing practically realizable algorithms. The theoretical study suggests that it is highly advantageous to exploit the size of the train by increasing the number of antennas, and further allowing the carriages to cooperate. In the practical system level study, we propose two low complexity MIMO-OFDM transmission schemes, which are based on simple antenna selection methods with spatial multiplexing. The main idea is to select the best transmit antennas among different antenna combinations by comparing their estimated throughput performances. The simulation results show that the proposed algorithms outperform LTE-based dynamic rank transmission schemes in terms of throughput and computational load in practical HST scenarios. Unlike the exhaustive search type of dynamic transmission schemes, our simple algorithms are also applicable to large antenna arrays. In conclusion, large antenna arrays with simple antenna selection and spatial multiplexing transmission strategies seem potential solutions to significantly improve the throughput of the base station to train link in HST scenarios.
\end{abstract}

Index Terms-Antenna selection, high mobility communications, MIMO-OFDM, spatial multiplexing, system level simulation.

\section{INTRODUCTION}

$\mathbf{T}$ HE increasing interest in the deployment of high speed trains (HSTs) in many parts of the world has evoked the need to provide high quality wireless communication services to onboard users. Following the recent trend in cellular communications, the HST passengers also need access to bandwidth hungry applications. However, providing high data rates and good quality of service (QoS) to passengers

This research was supported by the Finnish Funding Agency for Technology and Innovation (TEKES), Nokia Networks, Anite Telecoms, Huawei Technologies, Broadcom Communications Finland, and Elektrobit Wireless Communications.

The authors are with the Centre for Wireless Communications, University of Oulu, P.O. Box 4500, 90014 University of Oulu, Finland. (e-mail: forename.surname@ee.oulu.fi) in the presence of rapidly varying channel conditions is a challenging task [1]. An effective way to increase data rate is the use of multiple-input multiple-output (MIMO) and orthogonal frequency division multiplexing (OFDM) techniques [2]. However, to achieve the available performance gains, knowing the channel characteristics is highly important. Hence, to ensure reliable communication for passengers, knowledge about the different types of high speed railway environments is required to ensure proper communication network planning and to optimize transmitter and receiver designs. The HST railway environment possesses distinctive propagation characteristics and design opportunities. Hence, the transmission schemes tailored for the conventional cellular communication environments are likely to be suboptimal. The propagation characteristics of the railway environment have been observed in a number of studies through channel measurements and analyses of such environments [3]-[8]. Channel sounding for HST railway environment was examined in [9], where the authors' focus was on HST MIMO and coordinated multipoint (CoMP) channel measurements.

With the knowledge of the channel properties, providing reliable communication for passengers still remains a challenge due to the fast moving and well shielded carriages which causes severe Doppler frequency shift, high penetration loss, outdated channel feedback information, frequent and large handover processes, increased power consumption of passengers' mobile equipments and low data rates [10]. To overcome some of these problems, different authors have proposed various ways of efficiently providing wireless services to passengers [11]-[15]. The most promising concept is the use of a two hop network architecture, where the communication link is divided into a base station to train link (the backhaul link) and a train to onboard link (the access link). This network architecture enhances cellular coverage since vehicular penetration loss (VPL), which can be as high as $23 \mathrm{~dB}$ [16], can be eliminated. Also large simultaneous group handover processes and the passengers' high power consumption are significantly reduced with the two hop network architecture. Further studies in [17] and [18] have shown that even though there is a performance gain with the network architecture compared to direct transmission, there are still challenges and the main bottleneck is the backhaul link.

Studies in [19] try to tackle the frequent handovers experienced over the backhaul link, with the use of two antennas mounted at the front and rear of the train. When the train 
moves towards the cell edge, the front antenna executes handover while the rear antenna is still connected to the serving base station (BS). In [20], the problem of high handover failure probability was addressed with the use of coordinated multiple point transmission concept to achieve diversity gain with the HST receiving signals from two adjacent BSs when moving through overlapping areas.

MIMO spatial multiplexing in high speed railway communications has been studied in [21]-[23]. Studies in [21] analyzed the achievable MIMO capacity for high speed train with multiple antennas based on 3-D modeling of line-of-sight (LOS) channel. The authors show that spatial multiplexing gains can be achieved with a proposed multi-group multiantenna (MGMA) scheme, in which the capacity gain can be achieved by adjusting the weights among the MGMA arrays. On the other hand, the approach in [22], is to reconstruct the channel through the method of principal component analysis such that the correlation in the railway channel matrix is reduced. Hence, spatial multiplexing gains can be achieved. Reference [23] employs the selection of the appropriate antenna spacing to ensure that the channel response is not rank deficient. The impact of imperfect channel state information (CSI) on high mobility systems, examined in [24], shows that accurate estimation and tracking of the fast time-varying fading are critical to reliable operation. Also studies in [25] have shown that in many mobile scenarios, the upper bound of the achievable data rate is mainly defined by the feedback delay of the CSI. Due to the outdated and imperfect CSI, the throughput gains provided by conventional precoding methods may be marginal or even non-existent. Conceptually simple antenna selection methods implemented at the transmitter have been studied with different receive antenna combinations and transmission schemes [26]-[30]. The performance of transmit antenna schemes relies on the required feedback of information on selected antenna index/indices from the receiver to the BS. However, most of these studies only consider single antenna selection. In [28], multiple transmit antenna selection for spatial multiplexing was achieved by considering the detection order of an ordered successive interference cancellation (SIC) receiver with power control per antenna. Antenna selection for spatial multiplexing for practically realizable receivers was studied in [29] and [30]. The number of transmitted streams in these studies were fixed and a subset among all possible transmit antennas was chosen.

The aforementioned antenna selection schemes are designed from the perspective of conventional cellular systems. However, there are two major differences between an HST system (considering the BS to train backhaul link) and a conventional cellular system. The number of receiving antennas and the speed of the receiver can be both notably higher in the HST scenario. These characteristics suggest that large antenna arrays can be used to improve the throughput performance of an HST communication system via the achieved multiplexing gain. Moreover, the size of the train can be exploited to ease the spatial separability of the transmitted signals at the receiver side. Due to the high speed of the train, conventional precoding methods may not provide any practical performance gains. Instead, simpler spatial multiplexing methods with antenna selection schemes could be of practical interest. However, with large antenna arrays, adaptive transmission schemes may require extensive computational load, especially if the design is based on the exhaustive search type of adaptivity. The main aim of this paper is to address the aforementioned possibilities and challenges, and to study how the throughput performance can be increased in a realistic HST communication scenario. The contributions of this paper are introduced next.

In this paper, we focus on maximizing the downlink throughput of the BS to train link. Our analysis is built on MIMO-OFDM techniques in theoretical link level and practical system level scenarios. We first consider the throughput maximization problem in a theoretical single-cell MIMO-OFDM train scenario, and describe two convex optimization based transmission strategies. The objective of the theoretical study is to give fundamental insight into the throughput maximization problem in a simple train scenario rather than providing practically realizable transmission algorithms. The theoretical results demonstrate that notable gains can be obtained when the number of antennas is increased at the BS and train, and the carriages of the HST cooperate with each other. In the practical analysis, we first apply and evaluate the LTE-based closedloop and open-loop precoding with fixed and dynamic rank transmission schemes to a realistic system level HST scenario. Then we compare its performance with the exhaustive search scheme. After which we propose two low complexity transmission schemes that are based on simplified antenna selection methods with spatial multiplexing. These practical schemes can easily fit into the LTE-Advanced transmission structure. In the algorithms, the best transmit antennas are selected among a set of antenna combinations by comparing their estimated throughput at the train, and sending the information to the BS via a feedback link. The key idea of the algorithms is to provide performance close to the exhaustive search method, but with significantly reduced computational load (i.e., the number of throughput calculations). The simulation results imply that a promising practical solution to notably increase the backhaul link throughput in an HST scenario is to use low complexity antenna selection and spatial multiplexing techniques, and have large antenna arrays at the BSs and train. The main contributions of this paper are summarized as follows:

- Theoretical study of the throughput maximization optimization problem in a single-cell MIMO-OFDM train scenario with and without cooperation among carriages.

- Practical low complexity MIMO-OFDM transmission schemes, which are based on simple antenna selection methods with spatial multiplexing.

- Practical system-level performance and complexity evaluation of the proposed transmission schemes and LTEbased open-loop and closed-loop precoding methods.

The rest of this paper is organized as follows. In Section II, the system models for both the theoretical and practical scenarios are introduced. The throughput maximization algorithms for both cooperative and non-cooperative train scenarios are described for the theoretical system in Section III. LTEbased transmission strategies are applied to an HST scenario in Section IV. In Section V, the proposed low complexity antenna selection and spatial multiplexing methods are introduced. 
Section VI describes the simulators used for both theoretical and practical scenarios. The simulation results are presented in Section VII. A brief discussion on the simulation results is presented in Section VIII and the conclusion is provided in Section IX.

The following notations are carried out in this paper. Vectors and matrices are represented by boldface lower and upper case letters, respectively. The conjugate transposes of a matrix $\mathbf{H}$ and a vector $\mathbf{h}$ are denoted as $\mathbf{H}^{H}$ and $\mathbf{h}^{H}$, respectively.

\section{SySTEM MODEL}

We consider a train communication scenario focusing on the BS to train link. The train has multiple carriages, each equipped with a single moving relay node (MRN). The number of MRNs is denoted by $M$. Each MRN has an external antenna array with $N_{r}$ receive antennas that are assumed to be evenly spaced along the length of the corresponding carriage. The number of transmit antennas at the BS is $N_{t}$. The system supports MIMO-OFDM communications. Frequency domain is divided into $C$ subcarriers. The downlink communications between the BS and the train is analyzed in simple singlecell and realistic multi-cell systems to reflect theoretical and practical scenarios, respectively. For the clarity of presentation, separate signal models are presented for both scenarios. In all cases, we assume linear transmit/receive design strategies.

\section{A. Theoretical Single-Cell Scenario}

With a BS connected to $M$ MRNs, two modes of operation are derived. In non-cooperative mode, each MRN is performing reception independently and there is no form of cooperation among the MRNs. In cooperative mode, the MRNs can cooperate with each other and act as a large receive antenna array in order to aid data reception.

1) MRN Non-Cooperative Mode: The BS is transmitting data to all MRNs simultaneously while each MRN is performing reception independently at the train. This is illustrated in Fig. 1 without the connecting link among the MRNs. This scenario is conceptually similar to a point-to-multipoint MIMO-OFDM system. The downlink received signal vector $\mathbf{y}_{c, m} \in \mathbb{C}^{N_{r}}$ at the $m^{\text {th }}$ MRN for the $c^{\text {th }}$ subcarrier is given by

$$
\mathbf{y}_{c, m}=\sum_{m=1}^{M} \sum_{l=1}^{L_{m}} \mathbf{H}_{c, m} \mathbf{m}_{c, m, l} \mathbf{s}_{c, m, l}+\mathbf{n}_{c, m}
$$

where $\mathbf{H}_{c, m} \in \mathbb{C}^{N_{r} \times N_{t}}$ is the channel matrix between the BS and the $m^{\text {th }} \mathrm{MRN}, \mathbf{m}_{c, m, l} \in \mathbb{C}^{N_{t}}$ is the unnormalized precoding vector for the $l^{\text {th }}$ stream of the $m^{\text {th }}$ MRN, $\mathrm{s}_{c, m, l} \in \mathbb{C}$ denotes the corresponding data symbol and $\mathbf{n}_{c, m} \sim$ $\mathcal{C N}\left(0, N_{0} \mathbf{I}_{N_{r}}\right)$ is the additive complex white Gaussian noise vector with zero mean and $N_{0}$ variance per element. The number of data streams given to the $m^{\text {th }}$ MRN is denoted by $L_{m}$. The total transmit power of the $\mathrm{BS}$ is given by $P=\sum_{c=1}^{C} \sum_{m=1}^{M} \sum_{l=1}^{L_{m}}\left\|\mathbf{m}_{c, m, l}\right\|_{2}^{2}$. The received signal to interference plus noise ratio (SINR) of the $l^{\text {th }}$ stream at the $m^{\text {th }}$ MRN can be written as

$$
\Gamma_{c, m, l}=\frac{\left|\mathbf{w}_{c, m, l}^{H} \mathbf{H}_{c, m} \mathbf{m}_{c, m, l}\right|^{2}}{\left\|\mathbf{w}_{c, m, l}\right\|^{2} N_{0}+\sum_{q=1}^{M} \sum_{\substack{i=1 \\(i, q) \neq(l, m)}}^{L_{q}}\left|\mathbf{w}_{c, m, l}^{H} \mathbf{H}_{c, m} \mathbf{m}_{c, q, i}\right|^{2}},
$$

where $\mathbf{w}_{c, m, l} \in \mathbb{C}^{N_{r}}$ denotes the receive filter for the $l^{\text {th }}$ stream of the $m^{\text {th }}$ MRN.

2) MRN Cooperative Mode: The BS serves the whole train simultaneously and all MRNs can jointly perform reception, as depicted in Fig. 1. Due to cooperation among the MRNs,

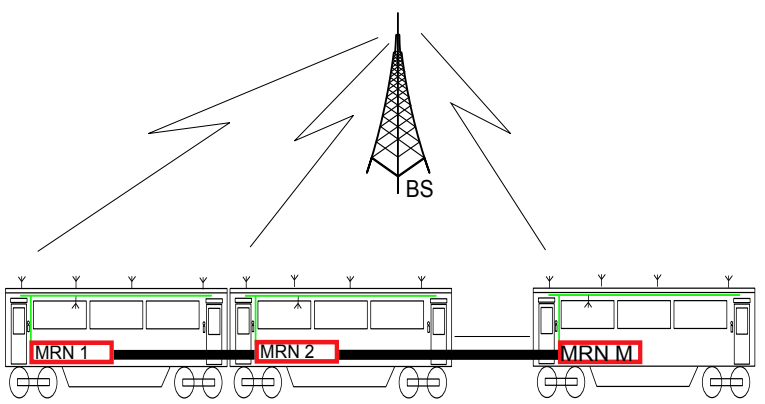

Fig. 1: Single-cell train scenario.

this scenario can be conceptually interpreted as a point-to-point MIMO-OFDM system. The signal model simplifies since all MRNs can be considered as a single receiver and the index of MRNs can be omitted. The downlink received signal vector $\mathbf{y}_{c} \in \mathbb{C}^{M N_{r}}$ for the $c^{\text {th }}$ subcarrier can be expressed as

$$
\mathbf{y}_{c}=\sum_{l=1}^{L} \mathbf{H}_{c} \mathbf{m}_{c, l} \mathbf{s}_{c, l}+\mathbf{n}_{c}
$$

where $\mathbf{m}_{c, l} \in \mathbb{C}^{N_{t}}$ denotes the precoding vector of the $l^{\text {th }}$ stream, $\mathrm{s}_{c, l} \in \mathbb{C}$ is the corresponding data symbol and $\mathbf{n}_{c} \in \mathbb{C}^{M N_{r}}$ is the additive complex white Gaussian noise vector. The total number of data streams transmitted to all $M$ MRNs is given by $L \leq \min \left(N_{t}, M N_{r}\right)$.The channel matrix from the BS to the whole MRN system is denoted by $\mathbf{H}_{c}=\left[\mathbf{H}_{c, 1}^{H} \ldots \mathbf{H}_{c, m}^{H} \ldots \mathbf{H}_{c, M}^{H}\right]^{H} \in \mathbb{C}^{M N_{r} \times N_{t}}$. The total transmit power is expressed as $P=\sum_{c=1}^{C} \sum_{l=1}^{L}\left\|\mathbf{m}_{c, l}\right\|_{2}^{2}$. Given the receive filter $\mathbf{w}_{c, l} \in \mathbb{C}^{M N_{r}}$ over all MRNs, the SINR for the $c^{\text {th }}$ subcarrier on the $l^{\text {th }}$ stream can be expressed as

$$
\Gamma_{c, l}=\frac{\left|\mathbf{w}_{c, l}^{H} \mathbf{H}_{c} \mathbf{m}_{c, l}\right|^{2}}{\left\|\mathbf{w}_{c, l}\right\|^{2} N_{0}+\sum_{\substack{i=1 \\ i \neq l}}^{L}\left|\mathbf{w}_{c, l}^{H} \mathbf{H}_{c} \mathbf{m}_{c, i}\right|^{2}} .
$$

\section{B. Practical Multi-Cell Scenario}

Fig. 2 provides a schematic representation of the HST in a multi-cell network configuration. The system consists of trisector antenna sites, where each sector forms a cell. The total number of cells in the system is $B$. For the ease of presentation, we refer to sectors as BSs in the rest of the paper. Each MRN can only have a direct connection to a single BS at a time. 


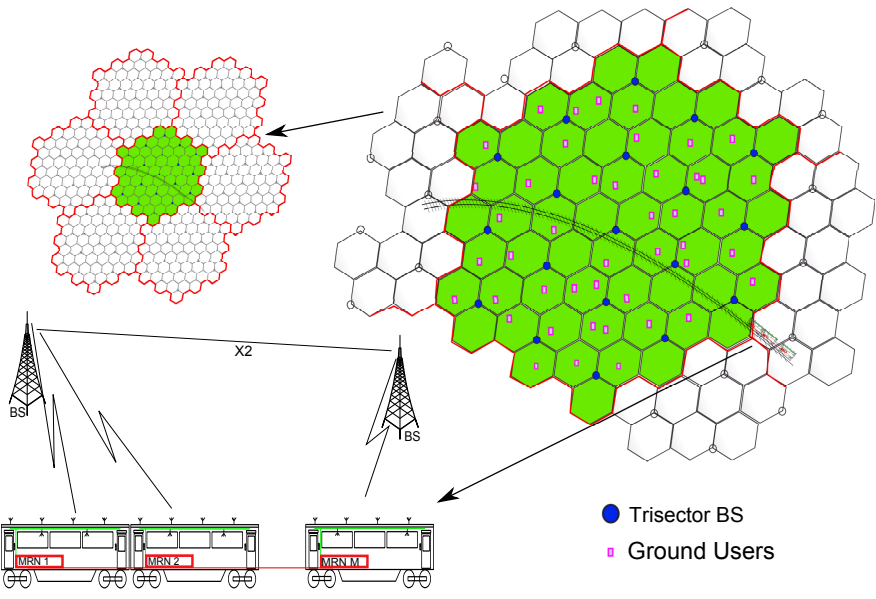

Fig. 2: Multi-cell train scenario.

In other words, a single MRN cannot be served by multiple BSs simultaneously. Different MRNs of the same HST can be connected either to the same BS or different BSs. Thus, an HST with multiple MRNs can be served by a single BS or multiple BSs, depending on the position of the HST in the network layout. The number of MRNs jointly associated to BS $b$ is denoted by $M_{b}$. Interference from onboard users to the MRNs' external antennas is omitted since half duplexing operation is assumed. We assume perfect synchronization since frequency offsets caused by the velocity of the HST can be compensated for [31] at different positions [32] along the predictable route despite the fast time varying Doppler shift. We also assume that $Q$ ground macro users with low mobility are randomly distributed in the multi-cell system and are therefore scheduled alongside the MRNs. Transmissions to ground users are considered as interference. We model the fast fading channel based on a geometry-based stochastic modeling approach, which allows the creation of an arbitrary double direction radio channel independent of any antenna configuration. Other channel parameters are based on statistical distributions extracted from real channel measurements and they are obtained stochastically [33]. More details on the used channel model are given in Section VI.

In this scenario, we consider MIMO-OFDM system operating in frequency division duplexing (FDD) mode and assuming that the MRNs served by the same BS can cooperate. The number of links formed depends on the number of BSs associated with the HST at every time instance. The MRNs coordinate within themselves and form groups based on MRNs associating with the same BS and each group formed cooperates as a single receive antenna array to establish a backhaul link. For clarity, we assume that the whole train is served by a single $\mathrm{BS}$ in the following signal model representation (i.e., $\left.M_{b}=M\right)$. The received signal vector $\mathbf{y}_{c} \in \mathbb{C}^{M N_{r}}$ for the MIMO transmission for subcarrier $c$ can be expressed as

$$
\mathbf{y}_{c}=\mathbf{H}_{c} \mathbf{M}_{c} \mathbf{s}_{c}+\sum_{k=1}^{K} \tilde{\mathbf{H}}_{c, k} \tilde{\mathbf{M}}_{c, k} \tilde{\mathbf{s}}_{c, k}+\mathbf{n}_{c}
$$

where $\mathbf{H}_{c} \in \mathbb{C}^{M N_{r} \times N_{t}}$ is the channel matrix between the serving $\mathrm{BS}$ and the entire MRN system, $\mathbf{M}_{c}=\left[\mathbf{m}_{c, 1} \ldots \mathbf{m}_{c, L}\right] \in$
$\mathbb{C}^{N_{t} \times L}$ is the unnormalized transmit precoding matrix for the desired data streams, $\mathbf{s}_{c} \in \mathbb{C}^{L}$ is the corresponding transmit signal vector and $\mathbf{n}_{c} \in \mathbb{C}^{M N_{r}}$ is the additive white Gaussian noise vector. Note that $\mathbf{M}_{c}$ can be expressed as $\mathbf{M}_{c}=\mathbf{P}_{c}^{1 / 2} \mathbf{F}_{c}$, where $\mathbf{F}_{c}=\left[\mathbf{f}_{c, 1} \ldots \mathbf{f}_{c, L}\right]$ is the normalized precoding matrix and $\mathbf{P}_{c}=\operatorname{diag}\left(P_{c, 1} \ldots P_{c, L}\right)$ consists of the powers allocated to each $L$ streams. $\tilde{\mathbf{H}}_{c, k} \in \mathbb{C}^{M N_{r} \times N_{t}}$, $\tilde{\mathbf{M}}_{c, k}=\left[\tilde{\mathbf{m}}_{c, 1} \ldots \tilde{\mathbf{m}}_{c, \tilde{L}_{k}}\right] \in \mathbb{C}^{N_{t} \times \tilde{L}_{k}}$, and $\tilde{\mathbf{s}}_{c, k} \in \mathbb{C}^{\tilde{L}_{k}}$ are the channel matrix from interfering BS $k$ to the MRN system, the precoder matrix used at BS $k$ to transmit $\tilde{L}_{k}$ (interfering) data streams and the corresponding signal vector. The total number of the interfering BSs is denoted by $K$.

The throughput optimal linear minimum mean square error (MMSE) filtering is applied at the receiver. The MMSE filter $\overline{\mathbf{W}}_{c}=\left[\overline{\mathbf{w}}_{c, 1} \ldots \overline{\mathbf{w}}_{c, L}\right] \in \mathbb{C}^{M N_{r} \times L}$ is obtained by minimizing the mean square error defined as $\mathbb{E}\left[\left\|\mathbf{s}_{c}-\overline{\mathbf{W}}_{c}^{H} \mathbf{y}_{c}\right\|^{2}\right]$, where

$$
\overline{\mathbf{W}}_{c}=\left(\hat{\mathbf{H}}_{c} \mathbf{M}_{c} \mathbf{M}_{c}^{H} \hat{\mathbf{H}}_{c}^{H}+\hat{\mathbf{R}}_{c}\right)^{-1} \hat{\mathbf{H}}_{c} \mathbf{M}_{c},
$$

where $\hat{\mathbf{H}}_{c}$ and $\hat{\mathbf{R}}_{c}$ are the estimates of the channel matrix $\mathbf{H}_{c}$ and the interference plus noise covariance matrix $\mathbf{R}_{c}$, respectively. The matrix $\mathbf{R}_{c}$ is given by

$$
\hat{\mathbf{R}}_{c}=\sum_{k=1}^{K} \hat{\mathbf{H}}_{c, k} \tilde{\mathbf{M}}_{c, k} \tilde{\mathbf{M}}_{c, k}^{H} \hat{\mathbf{H}}_{c, k}^{H}+N_{0} \mathbf{I}_{M N_{r}} .
$$

The SINR of transmission stream $l$ for subcarrier $c$ at the output of the MMSE receiver is given by

$$
\Gamma_{c, l}=\frac{\left|\overline{\mathbf{w}}_{c, l}^{H} \hat{\mathbf{H}}_{c} \mathbf{m}_{c, l}\right|^{2}}{\sum_{j=1, j \neq l}^{L}\left|\overline{\mathbf{w}}_{c, l}^{H} \hat{\mathbf{H}}_{c} \mathbf{m}_{c, j}\right|^{2}+\overline{\mathbf{w}}_{c, l}^{H} \hat{\mathbf{R}}_{c} \overline{\mathbf{w}}_{c, l}} .
$$

\section{PRECODING SCHEMES FOR TheORETICAL SCENARIO}

In this section, the throughput maximization problem is formulated for a downlink single-cell MIMO-OFDM train system with and without cooperation between MRNs. It is assumed that the channel is known at the BS and the MRNs. We propose precoding schemes that maximize the throughput given the aforementioned assumptions.

\section{A. MRN Non-Cooperative Mode}

In order to maximize throughput, the optimization problem of sum rate maximization under total transmit power constraint can be formulated as follows

$$
\begin{array}{ll}
\underset{\left\{\mathbf{m}_{c, m, l}, \mathbf{w}_{c, m, l}\right\}_{c=1, m=1, l=1}^{C, M, L}}{\operatorname{maximize}} & \sum_{c=1}^{C} \sum_{m=1}^{M} \sum_{l=1}^{L} \log \left(1+\Gamma_{c, m, l}\right) \\
\text { subject to } & \sum_{c=1}^{C} \sum_{m=1}^{M} \sum_{l=1}^{L}\left\|\mathbf{m}_{c, m, l}\right\|_{2}^{2} \leq P .
\end{array}
$$

Note that a positive weighting factor can be added to the objective function of (9) in order to maintain a certain degree of fairness among the MRNs, and possibly reflect the number of onboard users in each carriage required to be served. Problem (9) is non-convex, and thus, it cannot be solved in its current form. However, (9) can be reformulated and approximated 
to obtain an efficient (sub-optimal) solution where the objective function (i.e., sum rate) converges. However, global optimality cannot be guaranteed due to the non-convexity of the original problem. The proposed algorithm is an extension of the approach in [34] to a multi-carrier system. The idea of the algorithm is to divide (9) into precoder and receive filter design problems, which are solved alternatingly until the objective function converges. In other words, the precoders are optimized while the receive filters are fixed, and vice versa. The optimal receive filters per-subcarrier are obtained by using the linear MMSE criterion. This is given by

$$
\begin{array}{r}
\overline{\mathbf{w}}_{c, m, l}=\left(\sum_{m=1}^{M} \sum_{l=1}^{L} \mathbf{H}_{c, m} \mathbf{m}_{c, m, l} \mathbf{m}_{c, m, l}^{H} \mathbf{H}_{c, m}^{H}+\right. \\
\left.\mathbf{I}_{N_{r}} N_{0}\right)^{-1} \mathbf{H}_{c, m} \mathbf{m}_{c, m, l} .
\end{array}
$$

In order to solve the precoders, the sum rate maximization problem can be reformulated as a sum log-mean square error (MSE) minimization problem, expressed as

$$
\begin{array}{ll}
\underset{\left\{\mathbf{m}_{c, m, l}\right\}_{c=1, m}^{C, M}, m, l=1}{\operatorname{minimize}} & \sum_{c=1}^{C} \sum_{m=1}^{M} \sum_{l=1}^{L} \log \left(\epsilon_{c, m, l}\right) \\
\text { subject to } & \sum_{c=1}^{C} \sum_{m=1}^{M} \sum_{l=1}^{L}\left\|\mathbf{m}_{c, m, l}\right\|_{2}^{2} \leq P
\end{array}
$$

where $\epsilon_{c, m, l}$ is the MSE at the $m^{\text {th }}$ MRN for the $c^{\text {th }}$ subcarrier on the $l^{\text {th }}$ stream and the relation between the MSE and SINR [35] is expressed as

$$
\epsilon_{c, m, l}^{-1}=1+\Gamma_{c, m, l}
$$

With fixed receive filters, the sum log-MSE minimization problem is still a non-convex problem. Therefore, the transmit precoder design is reformulated using difference of convex function program (DCP) by introducing an auxiliary constraint

$$
\epsilon_{c, m, l} \leq 2^{-t_{c, m, l}}
$$

where the auxiliary variable $t_{c, m, l}$ is assumed to be such that $2^{t_{c, m, l}} \geq 1$. This implies that the domain of the convex MSE upper bounding function $2^{-t_{c, m, l}}$ is in the range of possible MSE values, i.e., between 0 and 1 . Note that also other exponential functions are applicable if they satisfy these assumptions. Applying (13) and relaxing the objective accordingly, the resulting problem is given by

$$
\begin{array}{ll}
\underset{\left.\mathbf{m}_{c, m, l}, t_{c, m, l}\right\}_{c}^{C, M, L}}{\operatorname{maximize}} & \sum_{c=1, m=1, l=1}^{C} \sum_{m=1}^{M} \sum_{l=1}^{L} t_{c, m, l} \\
\text { subject to } & \sum_{c=1}^{C} \sum_{m=1}^{M} \sum_{l=1}^{L}\left\|\mathbf{m}_{c, m, l}\right\|_{2}^{2} \leq P \\
& \epsilon_{c, m, l} \leq 2^{-t_{c, m}, l} \quad \forall c, m, l .
\end{array}
$$

The relaxation is tight since the inequality constraints achieve the optimal solution with equality. Problem (14) is still non-convex. Next, we apply successive convex approximation (SCA) method to approximate (14), and then, we iteratively solve the resulting convex problems such that the objective function converges. Now, a linear approximation of $f\left(t_{c, m, l}\right)=2^{-t_{c, m, l}}$ is found by taking the first order Taylor series approximation at a point $t_{c, m, l}^{(i)}$. This is expressed as

$$
\begin{aligned}
\bar{f}\left(t_{c, m, l}, t_{c, m, l}^{(i)}\right) & =f\left(t_{c, m, l}^{(i)}\right)+\left(t_{c, m, l}-t_{c, m, l}^{(i)}\right) f_{t_{c, m, l}}^{\prime}\left(t_{c, m, l}^{(i)}\right) \\
& =-a_{c, m, l}^{(i)} t_{c, m, l}+b_{c, m, l}^{(i)}
\end{aligned}
$$

where $f^{\prime}$ denotes the partial derivative and

$$
a_{c, m, l}^{(i)}=\ln (2) 2^{-t_{c, m, l}^{(i)}}, \quad b_{c, m, l}^{(i)}=2^{-t_{c, m, l}^{(i)}}\left(1+\ln (2) t_{c, m, l}^{(i)}\right) .
$$

As a result, we can formulate the following optimization problem at the $i$ th iteration of the SCA method for fixed $\left\{t_{c, m, l}^{(i)}\right\}_{c=1, m=1}^{C, M, L}$

$$
\begin{array}{ll}
\underset{\left.\mathbf{m}_{c, m, l}, t_{c, m, l}\right\}_{c=1, m=1, l=1}^{C, M, L}}{\operatorname{maximize}} & \sum_{c=1}^{C} \sum_{m=1}^{M} \sum_{l=1}^{L} t_{c, m, l} \\
\text { subject to } & \sum_{c=1}^{C} \sum_{m=1}^{M} \sum_{l=1}^{L}\left\|\mathbf{m}_{c, m, l}\right\|_{2}^{2} \leq P \\
& \epsilon_{c, m, l} \leq-a_{c, m, l}^{(i)} t_{c, m, l}+b_{c, m, l}^{(i)} .
\end{array}
$$

The next point of approximation $t_{c, m, l}^{(i+1)}$ can be found by using a line search method in [34]. The SCA-based convex problems with the updated approximation points are repeatedly solved. The objective value converges since it increases monotonically at each iteration of the SCA method and the receive filter update via the MMSE. The complete method is described in Algorithm 1.

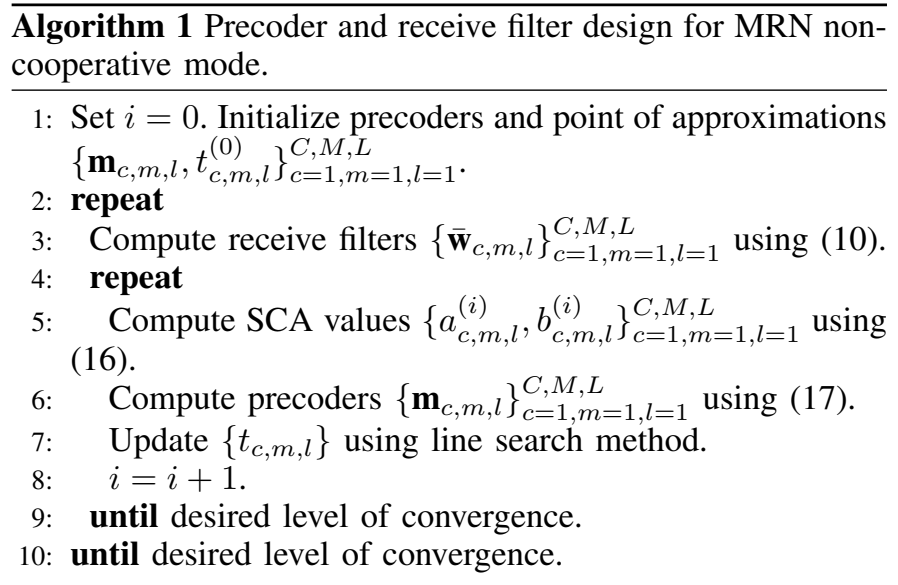

\section{B. MRN Cooperative Mode}

To maximize throughput, well known singular value decomposition (SVD) and water-filling principles can be exploited. Specifically, we apply SVD to each per-subcarrier channel 
matrix and use the resulting right singular vectors as precoders and left singular vectors as receive filters. This is written mathematically as $\mathbf{H}_{c}=\mathbf{U}_{c} \Lambda_{c} \mathbf{V}_{c}^{H}$, where the columns of $\mathbf{V}_{c} \in$ $\mathbb{C}^{N_{t} \times N_{t}}$ and $\mathbf{U}_{c} \in \mathbb{C}^{M N_{r} \times M N_{r}}$ are the right and left singular vectors, respectively. The diagonal entries of $\Lambda_{c} \in \mathbb{R}^{M N_{r} \times N_{t}}$ are the ordered singular values $\left(\lambda_{c, 1}, \ldots, \lambda_{c, L}\right)$ of $\mathbf{H}_{c}$ with the remaining entries equal to zero. The optimal power allocation is achieved by extending the MIMO water-filling algorithm in [36] to MIMO-OFDM systems, where the resulting waterfilling algorithm is performed over the independent MIMO channels and subcarriers. This transmission technique is also capacity achieving strategy. The overall transmitter-receiver strategy achieves the channel capacity as follows

$$
\begin{gathered}
\bar{C}=\sum_{c=1}^{C} \sum_{l=1}^{L} \log \left(1+\frac{P_{c, l}^{*}\left|\mathbf{u}_{c, l}^{H} \mathbf{H}_{c} \mathbf{v}_{c, l}\right|^{2}}{N_{0}}\right) \text {, with } \\
P_{c, l}^{*}=\left(\mu-\frac{N_{0}}{\left|\mathbf{u}_{c, l}^{H} \mathbf{H}_{c} \mathbf{v}_{c, l}\right|^{2}}\right)^{+}
\end{gathered}
$$

where the so-called water-level $\mu$ is chosen to satisfy the total power constraint, i.e., $\sum_{c=1}^{C} \sum_{l=1}^{L} P_{c, l}^{*}=P$. Symbols $\mathbf{v}_{c, l}$ and $\mathbf{u}_{c, l}$ denote the $l^{\text {th }}$ column of $\mathbf{V}_{c}$ and $\mathbf{U}_{c}$, respectively.

\section{LTE Precoding Schemes Applied to High Speed TRAIN SCENARIO}

This section examines the existing LTE precoding codebook schemes and how we apply them to the HST scenario for performance evaluation. The type of the codebooks used and the rationale behind the choice is briefly explained first. Precoding at the BS requires information about the MIMO channel. The channel information can be obtained via feedback from the MRNs. Unfortunately, the feedback of the channel information involves a significant overhead on the uplink capacity for most systems, especially on systems with high bandwidth and/or high mobility [37]. Thus, a necessary and practical solution is the use of limited feedback, where both the BS and radio terminal share a common codebook. The radio terminal searches the codebook for vectors/matrices that can maximize the overall system performance and the index of that vector/matrix is fed back to the BS. A combination of discrete fourier transform (DFT) based codebook [38] and householder $(\mathrm{HH})$ based codebook [39] is adopted by LTE and LTE-A due to its high precoding gain, lower feedback overhead, lower complexity and flexible support for various antenna configurations.

Based on the $\mathrm{HH}$ codebook design, we examine the impact of the LTE based closed-loop and open-loop spatial multiplexing schemes on the railway network. In the closed-loop spatial multiplexing scheme, the MRN/receiver estimates the channel feedback information, which includes the channel quality indicator (CQI), precoding matrix indicator (PMI), and rank indicator (RI) and feeds back the channel feedback information to the BS in order to maximize the spectral efficiency. The CQI value, which is based on the derived SINR, indicates the optimum modulation and coding scheme (MCS) to be used for the next transmission. The PMI chooses the optimum precoder matrix from a predefined codebook and the index of the chosen precoder matrix is conveyed to the BS. While the RI chooses the optimum number of layers for MIMO transmissions. In the case of the open-loop spatial multiplexing scheme, the estimated channel feedback information includes only the CQI and the RI. The feedback of the PMI is not required, however a subset of codebook is applied at the BS together with cyclic delay diversity (CDD). The open-loop pre-coding matrix is defined as

$$
\mathbf{F}_{c}=\mathbf{F}_{n}[i] \mathbf{D}_{c} \mathbf{U}
$$

where $\mathbf{F}_{n}[i] \in \mathbb{C}^{N_{t} \times L}$ is the $i$ th precoding matrix from the chosen subset of the $n$th codebook, $\mathbf{D}_{c}$ is a CDD matrix of size $L \times L$ that changes with the index of the sub-carriers, $\mathbf{U}$ is a fixed DFT matrix of size $L \times L$. The CDD $\mathbf{D}_{c}$ and the DFT $\mathbf{U}$ matrices are defined for 2, 3, and 4 transmission layers in [40], [41].

The selection criteria for the PMI and RI are based on maximizing the throughput, which is defined as

$$
T_{r, f}=\sum_{c=1}^{C} \sum_{l=1}^{r} \log _{2}\left(1+\Gamma_{c, l}\right)
$$

where the subscript $f$ signifies the selected precoder matrix and $r$, which can vary from 1 to $\leq \min \left(N_{t}, N_{r}\right)$, is the number of transmission rank/streams selected. The SINR $\Gamma_{c, l}$ is taken from (8). When fixed rank transmissions are considered $r=\min \left(N_{t}, N_{r}\right)$. The throughput maximizing PMI and RI values for the closed-loop scheme involves an exhaustive search through the codebook, which size grows exponentially with an increase in the number of transmit antennas. These values are chosen according to

$$
\left(r^{*}, f^{*}\right)=\arg \max _{r \in \mathcal{R}, f \in \mathcal{B}} T_{r, f}
$$

where $r^{*}$ is the selected transmission rank from the set $\mathcal{R}$, which consists of possible transmission ranks, i.e, $\mathcal{R}=$ $\left\{1, \ldots, \min \left(N_{t}, N_{r}\right)\right\}$. The index $f^{*}$ is the chosen precoder matrix from the codebook $\mathcal{B}$, which includes the predefined set of precoders for different transmission ranks. On the other hand, the throughput maximizing RI value in the open-loop spatial multiplexing scheme is chosen according to

$$
r^{*}=\arg \max _{r \in \mathcal{R}} T_{r, f}
$$

where $f$ is a precoder matrix selected in a predefined and deterministic way according to (19). It is worth noting that even if the transmission rank changes, all transmit antennas are always used for data transmissions.

\section{Practical Transmission SCHEMES Based ON Antenna Selection and Spatial Multiplexing}

In this section, two low complexity transmission schemes with simplified antenna selection and spatial multiplexing are proposed for the HST scenario. Before a detailed description, the rationale behind these schemes is given. There are two fundamental differences between a HST system (considering the BS to train backhaul link) and a conventional cellular system. The receiver speed and the number of receiving antennas can be both significantly higher in the HST scenario than in the traditional cellular system. Thus, designing practical transmission schemes for the HST scenario requires a change of 
perspective to start with. In LTE systems, the use of codebookbased precoding schemes can provide improved throughput performance in conventional cellular scenarios. However, in high mobility scenarios, such as HST, the achievable gains in throughput performance by using precoding may be minimal or even non-existent as compared with transmission strategies without precoding, as will be shown via numerical examples in Section VII. Moreover, the conventional LTE-based dynamic rank transmissions, where the number of data streams is adapted to the prevailing channel conditions, are mainly designed for mobile users with low number of receiving antennas. Using LTE-based precoding schemes with dynamic rank transmissions, the performance metric is calculated for all different precoder/stream combinations, and the one with the highest value is selected for the next transmission. This exhaustive search type of process may require extensive amount of computation since the number of different combinations can be high, especially while having large antenna arrays also at the receiver side.

In this respect, we propose two low complexity algorithms that are based on spatial multiplexing and simplified antenna selection. The algorithms are less complex and provide improved performance as compared with the LTE precoding schemes with dynamic rank transmissions. Furthermore, these low complexity algorithms are also applicable to the case where large number of antennas is used at the transmitter and receiver. The proposed practical transmission schemes are implemented at the receiver side of a communication link by utilizing the estimated MIMO channels using cooperative MRNs at the train. The information on the selected number and positions of the transmit antennas is fed back to the BS, which then performs data transmission using spatial multiplexing with the chosen set of antennas. In the used spatial multiplexing technique, precoding is not used, and each data stream is sent from a single transmit antenna. At the receiver side, each MRN performs the linear MMSE reception. Due to cooperation between the MRNs, all the MRNs connected to the same BS within a transmission time interval can be seen as a large MMSE receiver.

\section{A. Adaptive Transmission Scheme Based on Two-Phase An- tenna Selection}

The first practical algorithm has two phases for a proper selection of the transmit antennas. The target of the first phase is to select an appropriate number of transmit antennas to be used for the spatial multiplexing transmission. The second phase aims to select the proper antenna positions for the given number of antennas chosen in the first phase. In both phases, the appropriate options are chosen that provide the highest estimated throughput assuming spatial multiplexing transmission. There are different ways to estimate the throughput performance at the receiver side. Since the MMSE receiver is used, it is logical to calculate post-MMSE throughput by assuming spatial multiplexing transmission, as given by

$$
T_{N_{t}}=\sum_{c=1}^{C} \sum_{l=1}^{N_{t}} \log _{2}\left(1+\frac{P_{c, l}\left\|\mathbf{w}_{c, l}^{H} \hat{\mathbf{h}}_{c, l}\right\|^{2}}{N_{0}+\sum_{i \neq l}^{N_{t}} P_{c, i}\left\|\mathbf{w}_{c, i}^{H} \hat{\mathbf{h}}_{c, i}\right\|^{2}}\right),
$$

where $\mathbf{w}_{c, l}$ is the MMSE receiver for stream $l$ at subcarrier $c$, and it is obtained from (6). For the later usage, the MIMO channel at subcarrier $c$ is denoted by $\hat{\mathbf{H}}_{c}=\left[\hat{\mathbf{h}}_{c, 1}, \ldots, \hat{\mathbf{h}}_{c, N_{t}}\right]$. Equation (23) gives the total throughput over the subcarriers and transmission streams. The index $N_{t}$ denotes the number of transmit antennas used. Another possible performance metric is pre-MMSE throughput given by

$$
T_{N_{t}}=\sum_{c=1}^{C} \sum_{l=1}^{N_{t}} \log _{2}\left(1+\frac{P_{c, l}\left\|\hat{\mathbf{h}}_{c, l}\right\|^{2}}{N_{0}+\sum_{i \neq l}^{N_{t}} P_{c, i}\left\|\hat{\mathbf{h}}_{c, i}\right\|^{2}}\right) .
$$

Note that (23) is more accurate metric, whereas (24) is computationally less complex. It is worthwhile to mention that alternative performance metrics than throughput can also be used. One such metric can be composed by calculating received signal powers and correlation levels for different combinations of antenna counts and positions. This idea is described in [42].

The key idea of the algorithm is to significantly reduce the number of combinations, which need to be compared to each other. This is obtained by selecting only a subset of possible combinations to be compared with each other in both phases of the algorithm. The intuition here is that the most relevant combinations are chosen for the subsets to be compared with each other, and the less relevant ones are left out from the comparison. The sizes of the subsets can be considered as design parameters, and there is a complexity-performance trade-off. Here the complexity refers to computational load, which is defined as the number of throughput calculations. The larger the size of the subset is, the better the performance and the higher the computational load. However, choosing the subsets properly the computational load can be significantly reduced while providing performance close to exhaustive search, as will be demonstrated via numerical examples in Section VII. It is assumed that the train has higher or equal number of antennas than that of the serving BS. The proposed antenna selection based transmission scheme is summarized in Algorithm 2.

The predefined set of options $\mathcal{N}$ depends on the maximum number of transmit antennas $N_{t_{\max }}$ available and the level of complexity that can be accommodated. The basic idea behind downsizing the number of antenna options is to choose the combinations that will most probably provide the highest throughput performance. Given that the large size of the train can be utilized at the reception for the spatial separability of the transmitted streams, having high number of transmit antennas will most probably provide high throughput. In other words, there will be no point to check the combinations with low number of antennas, especially when the maximum antenna count is large. For example, antenna counts less than 10 may be omitted if the maximum number of transmit antennas is 20 . 
$\overline{\text { Algorithm } 2 \text { Two-phase antenna selection based adaptive }}$ transmission scheme for HST scenario.

1: Based on the MIMO channel estimate $\hat{\mathbf{H}}=\left[\hat{\mathbf{H}}_{1}, \ldots, \hat{\mathbf{H}}_{C}\right]$ at the train, select the best number of transmit antennas $N_{t}^{*}$ among a predefined set of options $\mathcal{N}$ by choosing the one with the highest calculated throughput using (23), i.e.,

$$
N_{t}^{*}=\arg \max _{N_{t} \in \mathcal{N}} T_{N_{t}} .
$$

2: Given the previously chosen number of transmit antennas $N_{t}^{*}$, select the best antenna positions $\mathcal{A}$ among a predefined set of combinations $\overline{\mathcal{A}}$ by choosing the one with the highest calculated throughput using (23), i.e.,

$$
\mathcal{A}^{*}=\arg \max _{\mathcal{A} \in \overline{\mathcal{A}}} T_{N_{t}(\mathcal{A})} \text {. }
$$

3: Send the index of the chosen antenna positions $\mathcal{A}^{*}$ to the BS.

4: At the BS, use spatial multiplexing with the chosen antenna positions $\mathcal{A}^{*}$ for data transmission.

5: At the train, estimate the MIMO channel $\hat{\mathbf{H}}$, and use the linear MMSE criterion for data reception. Go to step 1.

Thus, it appears to be beneficial to choose only high number of antennas for the first phase of the algorithm.

In this first phase, only one antenna combination is selected for each antenna count. This selection could be random or the antennas could be chosen such that they are as far apart as possible to each other in order to provide as good spatial separability as possible. In the second phase of the algorithm different antenna combinations of the chosen antenna count in the first phase are compared, and the best one chosen for the transmission. If the antenna count chosen in the first phase is close to maximum (e.g., 19 out of 20), the performance difference between different combinations in the second phase may be marginal since the corresponding radio channel characteristics are close to each other. Hence, it is unnecessary to compare all these combinations to each other in the second phase. The number of combinations can be reduced by randomly selecting only a few of them to be compared. In conclusion, the basic idea behind Algorithm 2 to provide high throughput performance with low computational load is to choose high antenna counts for the first phase and select only a few antenna combinations of them for the second phase. In general, most of the antenna counts and the corresponding combinations are not relevant in terms of throughput performance. This is emphasized when the maximum antenna count is high.

Intuitively speaking, the first phase may be more critical since the performance difference between the best and the worst choices is probably higher than that in the second phase. Note that the feedback overhead with Algorithm 2 implementation is comparable with the LTE dynamic transmission schemes, since the antenna combinations are predefined and known at the BS and HST. Hence, only the index of the antenna combinations will be required for the feedback. Algorithm 2 can be designed such that the computational load is (mostly) lower than the LTE open-loop transmission scheme, as will be demonstrated in Section VII.

\section{B. Adaptive Transmission Scheme Based on One-Phase An- tenna Selection}

The second practical algorithm selects an appropriate number of transmit antennas and the proper positions of the active antennas simultaneously for spatial multiplexing transmission. The algorithm operates as follows. The total throughput is first calculated for the maximum number of transmit antennas $N_{t_{\max }}$ using (23) or (24). Since spatial multiplexing transmission is assumed, different transmit antennas can be ordered based on their total throughput performance. The transmit antenna with the lowest throughput is removed, and the total throughput is calculated again for $N_{t_{\max }}-1$ transmit antennas. The two calculated throughput values are compared. If the first value is higher, the maximum number of transmit antennas is chosen for spatial multiplexing based data transmission. Otherwise, the throughput of $N_{t_{\max }}-1$ antennas is compared to the throughput calculated for $N_{t_{\max }}-2$ antennas when the second worst antenna is removed. The process of reducing the number of transmit antennas one after the other based on the lowest per transmit antenna throughput is repeated until the total throughput is decreased. In practice, if the total throughput difference between the current and previous case is relatively small, it may be worthwhile to continue the process until the performance difference is large enough. Therefore, there can be a predefined threshold $\alpha$ for the throughput difference, which needs to be exceeded to stop the process, and select the previous number and positions of the transmit antennas. The second antenna selection based transmission scheme is summarized in Algorithm 3. It will be shown in Section VII that Algorithm 3 has even lower computational load than that of Algorithm 2. However, the feedback overhead is somewhat higher since the antenna positions are not chosen from a predefined set of options.

\section{Simulator Description}

In this section, the theoretical single-cell and practical multi-cell simulators are introduced, and the main simulation parameters are presented.

\section{A. Theoretical Simulation model}

The theoretical simulation model consists of a single BS with $N_{t}$ transmit antennas serving a four-carriage HST. Each carriage is equipped with an MRN having $N_{r}$ receive antennas. The number of subcarriers is set to $C=10$. The SNR for the MRN is defined as $P / N_{0}$. Two different channel models are used to model NLOS scattering and LOS type of conditions with uncorrelated and correlated antennas, respectively. For the case of uncorrelated antennas, the Rayleigh fading channel model is exploited. In this model, each channel coefficient is drawn from a Gaussian distribution with zero mean and unit variance. For the correlated antennas, we have used the stochastic Weichselberger channel model [43]. In this case, the received signals for each path of the different antenna elements 
$\overline{\text { Algorithm } 3 \text { One-phase antenna selection based adaptive }}$ transmission scheme for HST scenario.

1: Based on the MIMO channel estimate $\hat{\mathbf{H}}=\left[\hat{\mathbf{H}}_{1}, \ldots, \hat{\mathbf{H}}_{C}\right]$ at the train, calculate the total throughput for the maximum number of transmit antennas $N_{t_{\max }}$ using (23). Set $N_{t}=$ $N_{t_{\max }}$.

2: Remove the antenna with the lowest throughput, and calculate the total throughput for $N_{t}-1$ antennas using (23). Compare the throughput values $T_{N_{t}}$ and $T_{N_{t}-1}$.

3: if

$$
T_{N_{t}}>T_{N_{t}-1}
$$

4: Stop, and choose $N_{t}$ transmit antennas and the corresponding antenna positions.

5: else

6: $\quad$ Set $N_{t}=N_{t}-1$, and go to step 2 .

7: end

8: Send the information of the chosen transmit antennas to the BS.

9: At the BS, use spatial multiplexing with the chosen transmit antennas for data transmission.

10: At the train, estimate the MIMO channel $\hat{\mathbf{H}}$, and use the linear MMSE criterion for data reception. Go to step 1.

are to be spatially correlated. Hence, spatial correlation matrices are generated to reflect the spatial correlation among the antenna elements. The spatial correlation coefficients for the BS and MRNs are generated according to

$R^{b}=\left(\begin{array}{cccc}1 & \rho_{1,2}^{b} & \cdots & \rho_{1, N_{t}}^{b} \\ \rho_{2,1}^{b} & 1 & \cdots & \rho_{2, N_{t}}^{b} \\ \vdots & \vdots & \ddots & \vdots \\ \rho_{N_{t}, 1}^{b} & \rho_{N_{t}, 2}^{b} & \cdots & 1\end{array}\right), R^{r}=\left(\begin{array}{cccc}1 & \rho_{1,2}^{r} & \cdots & \rho_{1, G}^{r} \\ \rho_{2,1}^{r} & 1 & \cdots & \rho_{2, G}^{r} \\ \vdots & \vdots & \ddots & \vdots \\ \rho_{G, 1}^{r} & \rho_{G, 2}^{r} & \cdots & 1\end{array}\right)$

where $G$ is defined as $G=N_{r}$ and $G=M N_{r}$ for MRN noncooperative mode and MRN cooperative mode respectively. The correlation coefficients are defined as

$$
\begin{gathered}
\rho_{i, j}^{b}=L_{i j} \mu, \quad \forall i=1, \ldots, N_{t} ; \quad l=1, \ldots, N_{t} \quad i \neq j \\
\rho_{k, l}^{r}=L_{k l} \mu, \quad \forall k=1, \ldots, G ; \quad l=1, \ldots, G \quad k \neq l
\end{gathered}
$$

where $\mu$ denotes a correlation factor generated randomly from a uniform distribution in the range $(0,1)$, while $L_{i j}$ and $L_{k l}$ are normalized path losses generated from the "Rural Macro" path loss model in [44]. The perpendicular distance between the BS and train, antenna spacing and mean angle of arrival (AoA) are taken into account in the calculation of the path losses. The mean AoA is derived from a uniform distribution in the range $(-\pi / 2, \pi / 2)$. The overall channel was modeled according to the Weichselberger model $H_{c}=U_{B S}\left(\Omega \odot H_{i i d}\right) U_{M R N}^{T}$ [43], which gives a better correlation structure than the Kronecker channel model [45]. $U_{B S}$ and $U_{M R N}$ are the orthonormal eigenvectors derived from the spatial correlation matrices for the BS and MRN, respectively. Symbol $\odot$ represents an element-wise multiplication and the coupling matrix $\Omega$, whose structure reflects the spatial arrangement of scattering objects, is derived from the eigenvalues of $R^{b}$ and $R^{r}$. In each simulation, the performance is averaged over 1000 independent channel realizations. The main simulation parameters for the case of correlated antennas are listed in Table I.

TABLE I: Simulation parameters for theoretical scenario (with correlated antennas).

\begin{tabular}{|c|c|}
\hline Parameters & Values \\
\hline Carrier frequency & $2 \mathrm{GHz}$ \\
\hline Number of channel realization & 1000 \\
\hline BS antenna spacing & 0.5 wavelength \\
\hline Length of each carriage & $40 \mathrm{~m}$ \\
\hline$\perp$ distance $(\mathrm{BS} \rightarrow$ HST) & $70 \mathrm{~m}$ \\
\hline MRN antenna spacing & Length of carriage/number of MRN antennas \\
\hline Number of BS/MRN antennas & $4,8,12,16$ \\
\hline correlation factor & Uniform distribution $(0,1)$ \\
\hline Angle of arrival & Uniform distribution $(-\pi / 2, \pi / 2)$ \\
\hline
\end{tabular}

\section{B. Practical Simulation Model}

The practical HST simulation model is based on a realistic wrap around multi-cell environment with a central layout of 57 cells (19 tri-sector antenna sites). This central layout is wrapped around with the copies of it to ensure uniform interference levels across the 57 cells, as shown in Fig. 2. The simulation run consists of 1000 drops with 50 channel samples per drop. 100 ground users are randomly distributed throughout the central layout at the start of each drop. The rail is laid across the central layout with a radius of $5 \mathrm{~km}$ such that the closest distance to any BS along the rail is 50 $\mathrm{m}$. The movement of the HST is modeled such that the HST moves incrementally along the rail at the start of each drop. The ground users and MRNs on the HST are paired with 57 cells providing the strongest received signal strength, which is calculated based on distance dependent pathloss and angular antenna gain. The pairings remain constant throughout each drop, therefore handover processes are not considered as such. MIMO-OFDM system operating in FDD mode is considered with a channel bandwidth of $10 \mathrm{MHz}$ according to the LTE standards [46].

At every transmission time interval (TTI), the resource scheduler at the BS distributes available resources among the active ground users and MRNs such that the overall throughput is maximized and the resources are fairly distributed. The resources are shared based on a modified proportional fair scheduling algorithm. First, the scheduler differentiates ground users from MRN and then splits the resources between ground users and MRN backhaul links based on the number of active ground users and MRNs. A maximum of 50 percent of the resources are made available to the BS to train backhaul links due to the half duplex operation of the MRN. Scheduling relies on channel information feedback, which may include CQI, PMI, RI and the proposed antenna selection indexing. Following the LTE framework, the channel feedback information is obtained by the receiver/MRN and is made available to the scheduler with a periodicity of $6 \mathrm{~ms}$ interval and $2 \mathrm{~ms}$ delay. The values of the feedback periodicity and delay depend on factors such as the granularity of the channel feedback information subband reporting, the capability of user equipment, and higher layer message (e.g., radio resource control 
(RRC) Connection Reconfiguration, RRC Connection Setup) [46]. The calculated channel feedback information is based on the channel coefficients made available at the receiver/MRN. Random errors are introduced into the channel coefficients before calculating the channel feedback information, to model channel estimation errors as no specific channel estimator was implemented. The channel coefficients are generated based on the WINNER II channel model [33]. It is a geometry based model that enables the separation of propagation parameters and antennas. Some of the parameters needed for the channel generation include the propagation scenario, the speed and direction of the UE, number, height, and location of the BS and MS/MRN, antenna field patterns, system centre frequency, etc. Based on the delayed channel feedback information, a modulation and coding scheme (MCS) is set for each terminal at the BS for the next transmission.

A link to system (L2S) level interface is used to map the link level SINR values to the system level throughput performance. More precisely, the obtained SINRs are mapped to mutual information values using mutual information effective SINR metric (MIESM) link layer abstraction [47]. The MCS values determine the frame error probability (FEP) at the link to system level interface and define the transport block size, i.e., the number of bits transmitted. The success of transmissions is identified by hybrid automatic repeat request (HARQ) acknowledgements, which are determined in the system level interface and fed back to the BS after a delay. The number of bits transmitted for successful transmissions and retransmissions are used in the throughput calculations. The described system level simulator is LTE compliant. The simulation parameters are set according to the guidelines established by the international telecommunications union radiocommunication sector (ITU-R) for international mobile telecommunications advanced (IMT-A) radio interface evaluation [48]. The main simulation parameters are listed in Table II.

TABLE II: Simulation parameters for practical scenario.

\begin{tabular}{|c|c|}
\hline Parameters & Values \\
\hline Carrier frequency & $2 \mathrm{GHz}$ \\
\hline System bandwidth & $10 \mathrm{MHz}$ \\
\hline Transmit power & $46 \mathrm{dBm}$ \\
\hline Number of ground users & 100 \\
\hline Number of MRNs & 8 (1 in each carriage) \\
\hline Propagation scenario & suburban macro/rural moving networks \\
\hline Inter-site distance (ISD) & $1.3 \mathrm{~km}$ \\
\hline Traffic Model & Full buffer \\
\hline MRN duplex mode & half duplex FDD \\
\hline Length of each carriage & $30 \mathrm{~m}$ \\
\hline Transmission scheme & spatial multiplexing \\
\hline HARQ & chase combining \\
\hline HARQ processes & 6 \\
\hline Receiver type & MMSE \\
\hline L2S interface metric & MIESM \\
\hline Train speed & $300 \mathrm{~km} / \mathrm{h}$ \\
\hline
\end{tabular}

VII. Simulation Results

In this section, the performance of different MIMO-OFDM transmission schemes is evaluated via numerical examples in

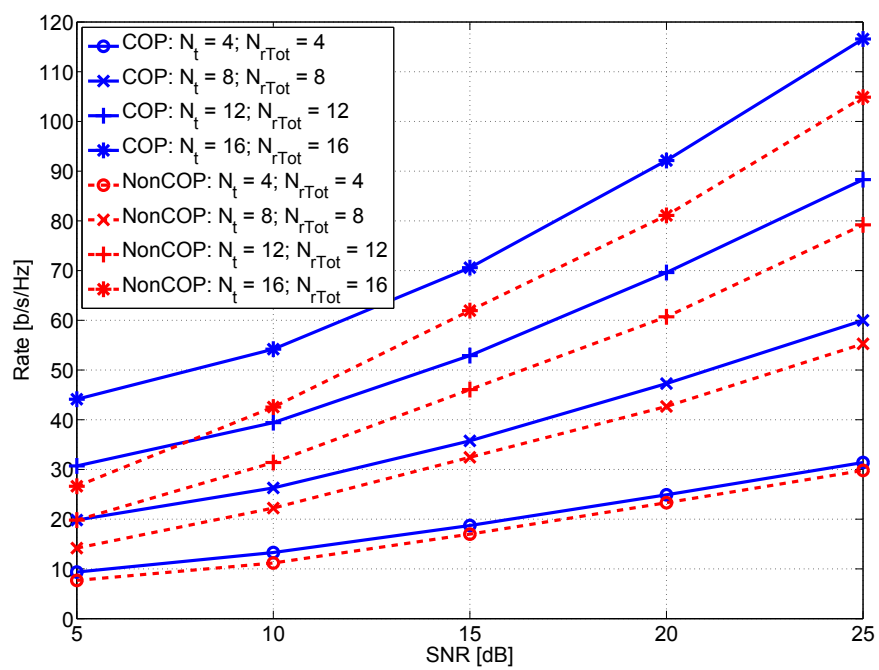

(a) i.i.d channel

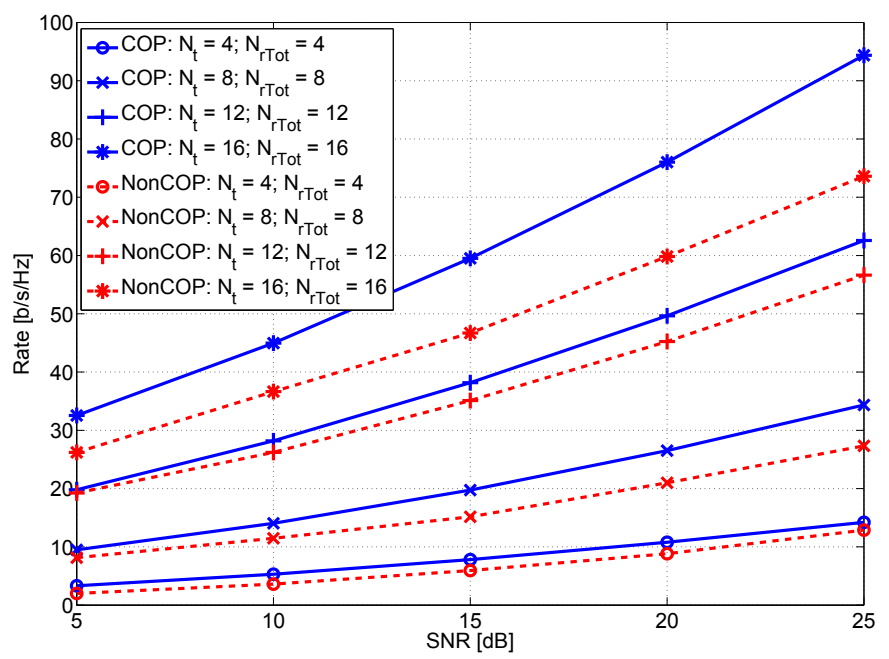

(b) Correlated channel

Fig. 3: Achieved sum rate for cooperative and non-cooperative MRN system.

theoretical and practical simulation models. First, we compare the performance of the cooperative and non-cooperative MRN systems with the increasing number of transmit and receive antennas in a simple single-cell scenario. Then, the performance of the LTE-based precoding schemes and the proposed simplified antenna selection algorithms are compared in a practical HST scenario. Finally, we study how much the throughput performance can be improved by increasing the number of transmit and receive antennas in the practical scenario.

In Fig. 3, the achieved sum rate is plotted against SNR for the cooperative (COP) and non-cooperative (NonCOP) MRN systems with different numbers of transmit and receive antennas in uncorrelated and correlated channels. The total number of receive antennas at the train is denoted by $N_{\mathrm{rTot}}=M N_{r}$. The simulation results show that the cooperation of MRNs is highly beneficial compared to non-cooperative scheme at all SINR values. The performance gain increases with the increas- 
ing number of transmit and receive antennas. The gains are even higher in the correlated channel conditions, even though the absolute performance is somewhat decreased compared to the uncorrelated channels due to the reduction of spatial degrees of freedom. The superior performance of cooperative scheme is due to a fact that the overall MIMO channel from the BS to the entire train can be divided into parallel interferencefree sub-channels with proper transmit and receive processing through the SVD. In the non-cooperative scheme with the independent data reception of each MRN, inter-MRN and inter-stream interferences limit the throughput performance. In other words, the spatial separability of the transmitted signals at the receiver side is more efficient for the cooperative scheme since it can exploit all the receive antennas and the physical length of the train. In conclusion, the theoretical results imply that the MRNs in the train should cooperate if possible and the antenna arrays at the BS and the train should be as large as practically possible. Hence for the rest of the paper, we only consider the cooperative scheme.

Before examining the performance analysis for different transmission schemes, we show the impact of different train speeds on the throughput of the cooperative scheme. Fig. 4

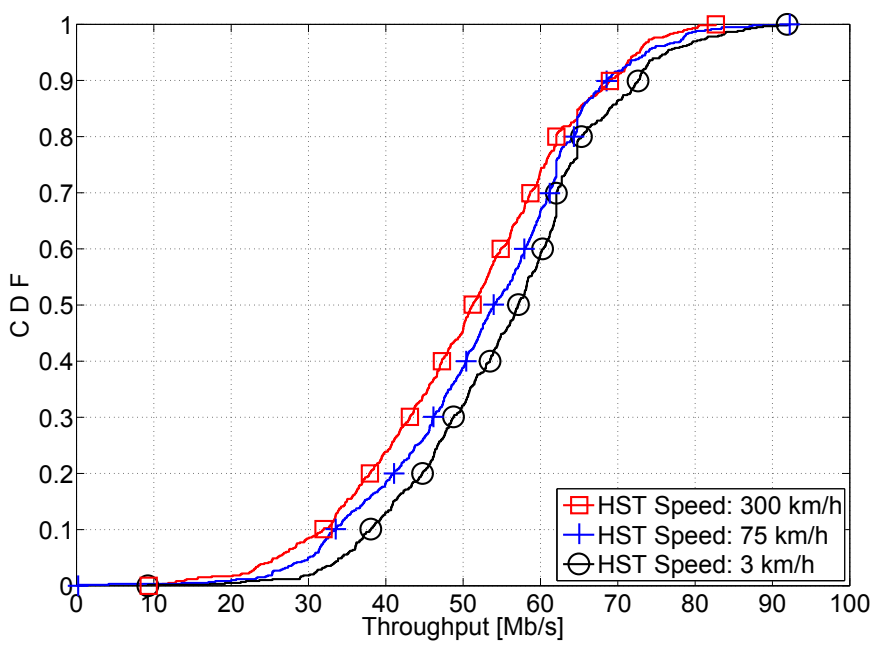

Fig. 4: CDF plot of throughput for different HST speed.

shows the cumulative distribution function (CDF) of the throughput for three different train speeds with antenna configuration of $N_{t}=4$ and $N_{r}=2$ for each of the MRN. The results show the trend that throughput decreases as the speed increases. The difference in the throughput is mainly a result of the impact of the delayed feedback of the channel information w.r.t. the channel and speed of the HST. ${ }^{1}$ Due to high speed, feedback information gets outdated fast.

In all the following simulations, the total transmit power of a BS is assumed fixed (i.e., $46 \mathrm{dBm}$ ) and this power is equally divided among the transmit antennas, regardless of the number of antennas used. Fig. 5 illustrates the CDF of the throughput

\footnotetext{
${ }^{1}$ The effect of Doppler shift and handover at different vehicular speeds were examined in [49].
}

for different transmission schemes at $300 \mathrm{~km} / \mathrm{h}$ train speed. The following transmission schemes are considered

- Spatial multiplexing: fixed rank (S-MUX)

- LTE closed-loop: fixed rank (LTE CL)

- LTE open-loop: fixed rank (LTE OL)

- LTE closed-loop: dynamic rank (LTE CL-dynamic rank)

- LTE open-loop: dynamic rank (LTE OL-dynamic rank)

- Spatial multiplexing: exhaustive search over all possible antenna combinations (S-MUX exhaustive search).

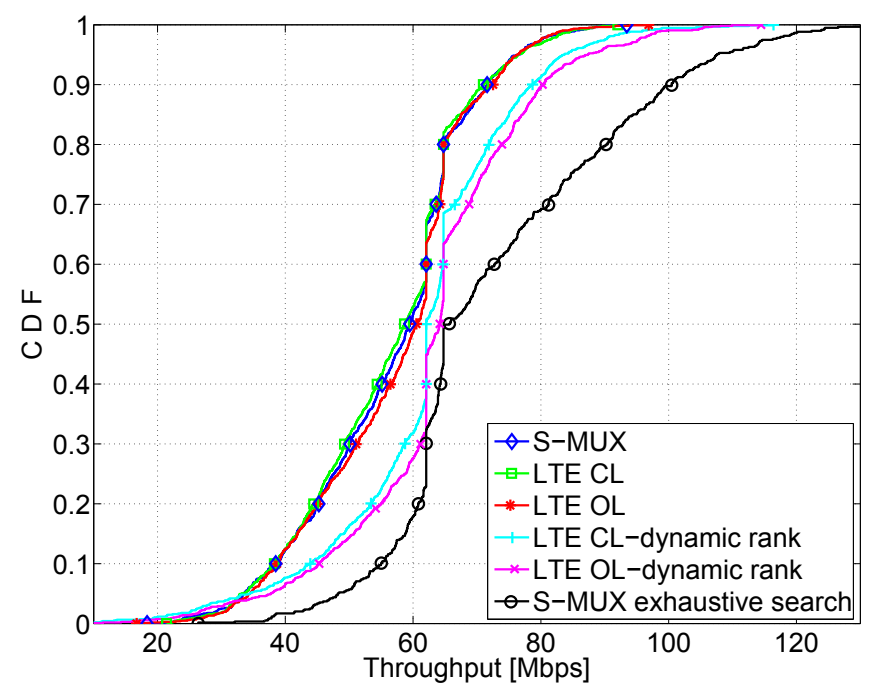

Fig. 5: CDF plot of throughput for fixed and adaptive transmission schemes with $N_{t}=4$ and $N_{r}=4$.

The results show that all the fixed rank transmission schemes have nearly comparable performance, regardless of the precoding. Thus, precoding-based schemes hardly provide any gain over the S-MUX. However, the dynamic rank transmissions provide significant performance gains as compared with the fixed rank schemes. It can be seen that the LTE OL-dynamic rank scheme slightly outperforms the $\mathrm{CL}$ one. The superior throughput performance can be achieved by using the SMUX with exhaustive search. The results imply that using precoding may not provide any gains in a practical HST scenario. However, significant gains seem to be available by using adaptive transmission schemes.

In Fig. 6, the CDF throughput performance of the proposed antenna selection (AS) algorithms is compared with the LTE OL-dynamic rank and the S-MUX exhaustive search transmission schemes. The proposed algorithms are represented as

- Alg.2 (2-phase AS)

- Alg.3 (1-phase AS)

As can be seen, the proposed algorithms achieve almost comparable performance to that of the S-MUX with exhaustive search, yet with significantly reduced computational load. The results also show that the proposed algorithms provide some performance gains when compared with the LTE OL-dynamic rank transmission scheme.

The computational loads involved for performing different 


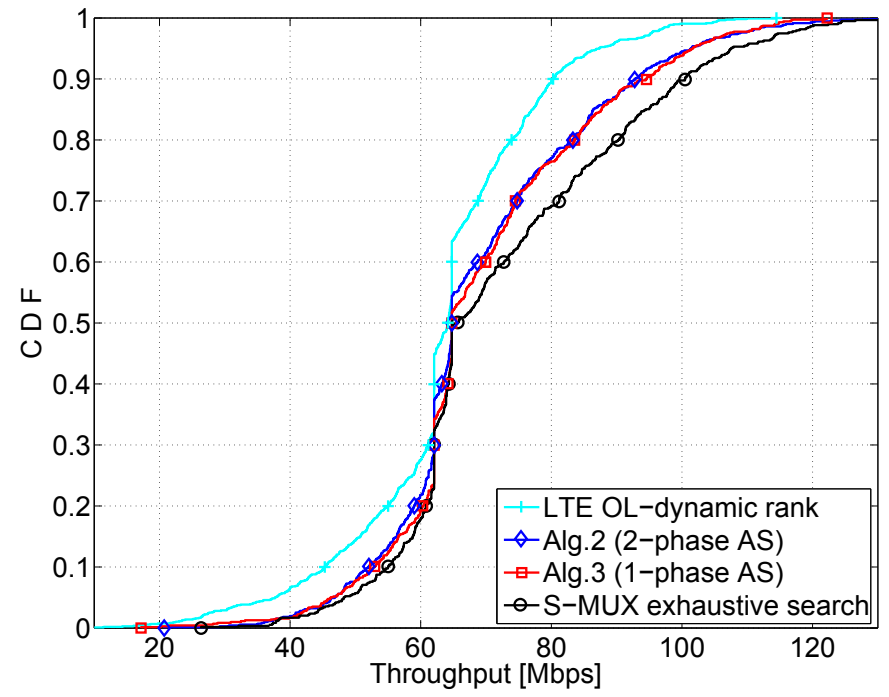

Fig. 6: CDF plot of throughput for adaptive transmission schemes with $N_{t}=4$ and $N_{r}=4$.

adaptive transmission schemes are evaluated in Table $\mathrm{III}^{2}$ in terms of per-stream throughput calculations per-subcarrier.

TABLE III: Number of per-stream throughput calculations.

\begin{tabular}{|l|l|l|l|l|l|}
\hline & \multicolumn{5}{|c|}{ Number of throughput calculations } \\
\hline$N_{t}$ & $\mathbf{4}$ & $\mathbf{8}$ & $\mathbf{1 2}$ & $\mathbf{1 6}$ & $\mathbf{2 0}$ \\
\hline LTE CL-dynamic rank & 144 & 905 & N/A & N/A & N/A \\
\hline LTE OL-dynamic rank & 9 & $35^{*}$ & N/A & N/A & N/A \\
\hline S-MUX exhaustive search & 28 & 1016 & 24564 & 524272 & 10485740 \\
\hline Alg.2 (2-phase AS) & 9.7 & 28.3 & 60.8 & 105.1 & 162.5 \\
\hline Alg.3 (1-phase AS) & 7.9 & 21 & 35.3 & 54 & 73 \\
\hline
\end{tabular}

Note that in the case of the proposed algorithms, the average per-stream throughput needs to be calculated over the simulation runs since the number of throughput calculations is not predefined, as in the case of all other schemes. For all the transmission schemes, single layer transmissions were omitted.

One can see that the proposed simple algorithms require significantly lower computational load than the S-MUX exhaustive search and CL-dynamic rank schemes. It is worthwhile to mention that one per-stream throughput calculation of a S-MUX-based scheme requires less computational load as compared with that of the precoding-based scheme. Nevertheless, this is not taken into account in Table III. The computational load of the proposed algorithms are slightly less than that of the OL-dynamic rank scheme except for Algorithm 2 with $N_{t}=4$, in which the complexities are comparable. However, the difference is increasing as the number of antennas increases. The number of throughput calculations is not presented for the LTE-based schemes when $N_{t}>8$

\footnotetext{
${ }^{2}$ Note that 8 transmit antennas is supported in LTE specification for LTE CL but not for LTE OL. However, computation for * LTE OL-dynamic rank as shown in Table III is based on a straightforward extension from the 4 transmit antennas.
}

since the LTE standard only support $N_{t} \leq 8$, and there are no codebooks defined for the higher number of antennas. It is worth mentioning that the predefined antenna combinations in the first and second phases of Algorithm 2 were chosen by following the principles described in Section $\mathrm{V}$ with the aim of keeping the computational load to a reasonable level. In the first phase, the number of antenna combinations $|\mathcal{N}|$ is related to the total number of transmit antennas, and is given by $|\mathcal{N}|=\left(N_{t_{\max }} / 2\right)-1$. The number of subsets in the predefined set of antenna combinations $\overline{\mathcal{A}}$ in the second phase ranges from 1 to 3 and the choice of subset depends on the antenna count chosen in the first phase relative to the total number of antennas. As an example, Table IV presents the predefined antenna combinations for the case of $N_{t_{\max }}=12$. The numbers in the brackets refer to the indexes of the used antennas.

TABLE IV: Predefined antenna combinations for Algorithm 2.

\begin{tabular}{|l|l|l|}
\hline Antenna count & 1st phase & 2nd phase \\
\hline 12 & $\{1,2,3,4,5,6,7,8,9,10,11,12\}$ & - \\
\hline 11 & $\{1,2,3,4,5,6,7,8,9,10,12\}$ & $\{1,3,4,5,6,7,8,9,10,11,12\}$ \\
\hline 10 & $\{1,2,3,4,5,6,7,9,11,12\}$ & $\{1,3,4,5,6,8,9,10,11,12\}$ \\
\hline 9 & $\{1,2,3,4,5,6,8,10,12\}$ & $\{1,3,4,5,7,8,9,11,12\}$ \\
& & $\{2,3,4,6,7,8,9,10,11\}$ \\
\hline 8 & $\{1,2,3,5,7,9,11,12\}$ & $\{1,3,4,6,7,8,10,12\}$ \\
& & $\{2,4,6,8,9,10,11,12\}$ \\
& & $\{1,3,5,7,8,9,11,12\}$ \\
\hline
\end{tabular}

Inspired by the theoretical simulation results, we next examine how much the throughput performance can be improved by increasing the number of transmit and receive antennas in a practical HST scenario. Fig. 7 shows the CDF of throughput performance for the S-MUX with fixed transmission scheme at various transmit and receive antenna array sizes. One can see that the performance significantly improves with the increasing number of antennas. However, the performance gains are getting somewhat smaller when the number of antennas is high, especially for the low percentile region. This is mainly due to a fact that increasing the number of antennas on the HST will reduce the antenna spacing among the antennas, thereby causing an increase in spatial correlation of the channel. Thus, the spatial separability of the transmitted streams becomes poorer.

The CDF throughput performance for the proposed antenna selection algorithms using the same number of transmit and receive antennas as in Fig. 7 is shown in Fig. 8. Comparing Fig. 7 to Fig. 8, the proposed algorithms show significant throughput performance gains. The percentage increase in the throughput at different CDF points compared to fixed S-MUX transmission scheme is illustrated in Table V. Algorithm 2 and 3 have comparable performances in most cases. Algorithm 3 has less computational load compared to Algorithm 2, however the feedback overhead is higher than in Algorithm 2 implementation. This is because the BS does not have prior knowledge of which transmit antennas are active or inactive in Algorithm 3 implementation. S-MUX exhaustive search could not be simulated because of the large number of throughput calculations, which grows exponentially with an increase in the number of antennas. The results imply that there are 


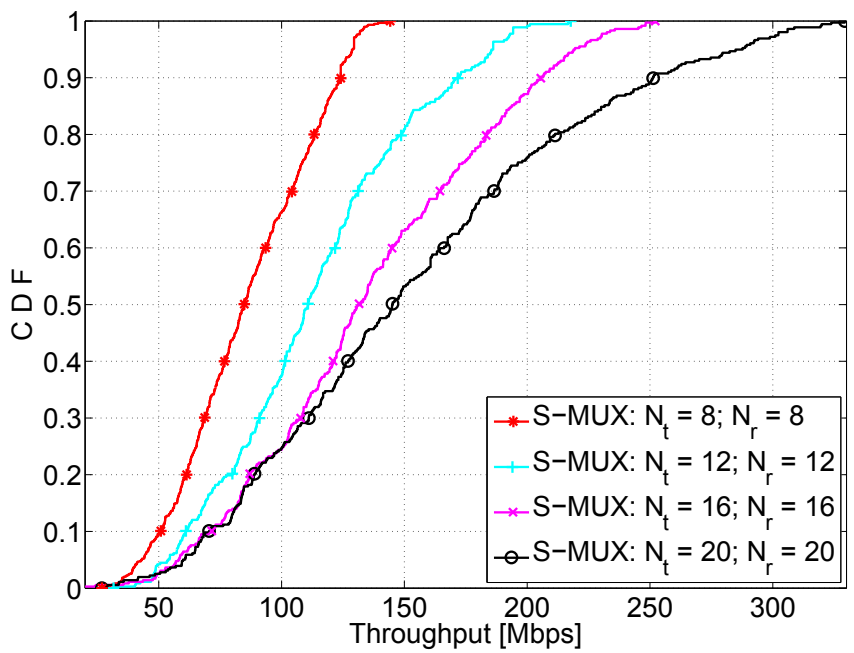

Fig. 7: CDF plot of throughput for fixed S-MUX transmission with increasing number of antennas.

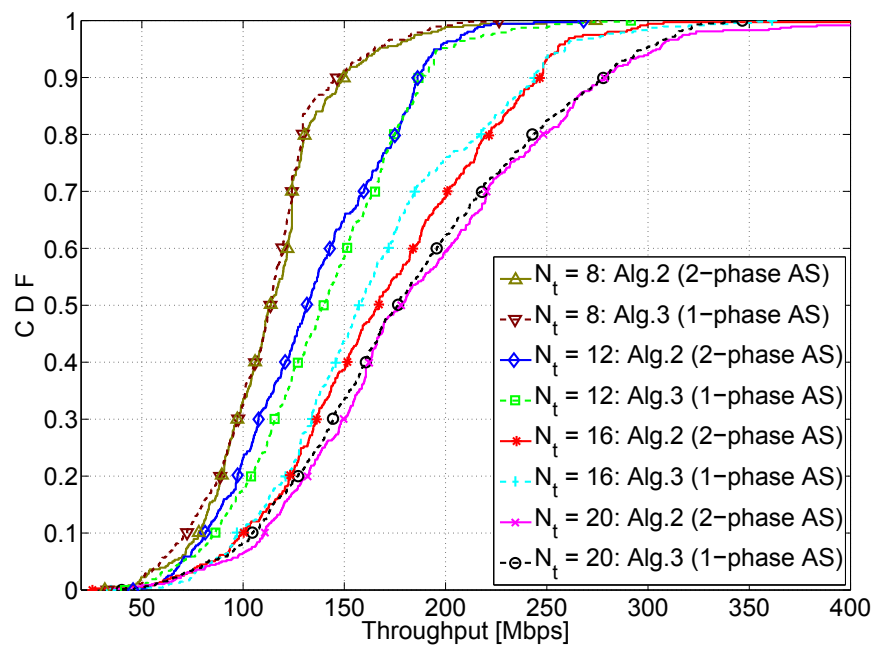

Fig. 8: CDF plot of throughput for the proposed adaptive transmission schemes with increasing number of antennas.

significant throughput gains available by increasing the number of antennas and using the proposed low complexity algorithms.

\section{DISCUSSION}

The simulation results imply that the key point for improving the throughput performance in a HST scenario is to use sufficiently large antenna arrays at the BS and train. The results also demonstrate that precoding is somewhat irrelevant, but transmission rank adaption via exhaustive search type of process is highly beneficial. However, rank adaptation requires a lot of computation, especially when using large antenna arrays. The key insight in reducing the computational load of the system is to simplify the exhaustive search process by comparing only the most relevant combinations, i.e., focusing on choosing the right number of transmit antennas rather
TABLE V: Performance gains of the proposed adaptive algorithms over fixed S-MUX scheme.

\begin{tabular}{|l|l|l|l|l|l|l|l|l|l|l|}
\hline & \multicolumn{7}{|c|}{ \% increase in throughput performance compared to S-MUX } \\
\hline CDF & \multicolumn{3}{|c|}{ Alg.2 (2-phase AS) } & \multicolumn{5}{c|}{ Alg.3 (1-phase AS) } \\
\hline$N_{t}$ & 4 & 8 & 12 & 16 & 20 & 4 & 8 & 12 & 16 & 20 \\
\hline 0.2 & $32 \%$ & $45 \%$ & $22 \%$ & $42 \%$ & $48 \%$ & $35 \%$ & $44 \%$ & $30 \%$ & $39 \%$ & $42 \%$ \\
\hline 0.5 & $6.3 \%$ & $34 \%$ & $19 \%$ & $27 \%$ & $22 \%$ & $6.3 \%$ & $34 \%$ & $26 \%$ & $19 \%$ & $22 \%$ \\
\hline 0.7 & $17 \%$ & $20 \%$ & $22 \%$ & $22 \%$ & $18 \%$ & $17 \%$ & $20 \%$ & $25 \%$ & $12 \%$ & $17 \%$ \\
\hline 0.9 & $30 \%$ & $21 \%$ & $9 \%$ & $19 \%$ & $11 \%$ & $30 \%$ & $17 \%$ & $9 \%$ & $18 \%$ & $11 \%$ \\
\hline
\end{tabular}

than going through all the possible combinations. It was shown that the proposed low complexity algorithms including spatial multiplexing with simplified antenna selection provide performance almost comparable with that of the exhaustive search scheme, but with notably reduced computational load. In conclusion, large antenna arrays at the transmitting and receiving sides, with simple spatial multiplexing and antenna selection transmission scheme seem an appropriate solution to significantly improve the backhaul link throughput in a practical HST communication scenario. These simplified algorithms also fit in well to real-time demanding scenarios such as the high speed moving receivers, where the time taken to select an appropriate transmission scheme is significantly reduced as a result of the reduced computational load.

\section{CONCLUSION}

This paper considered a MIMO-OFDM HST communication system where the focus was on the backhaul link optimization with the aim of throughput maximization. This problem was approached from both theoretical and practical aspects. The theoretical results showed that cooperation between the MRNs and increasing the number of antennas is highly beneficial. In a practical HST scenario, it was shown that adaptive rank transmission schemes provide significant throughput gains over fixed rank schemes. However, adaptive schemes may require extensive amount of computation, especially when the number of antennas is high. To significantly reduce the computational load, we proposed two low complexity transmission schemes with simplified antenna selection and spatial multiplexing. The practical simulation results demonstrated that the proposed simple algorithms provide almost similar performance as compared with the spatial multiplexing with exhaustive search and improved throughput performance as compared with the LTE-based dynamic rank schemes. Furthermore, computational load was significantly reduced. It was also shown that the throughput is further improved by combining the proposed low complexity algorithms with large antenna arrays.

\section{REFERENCES}

[1] X. Zhu, S. Chen, H. Hu, X. Su, and Y. Shi, "TDD-based mobile communication solutions for high-speed railway scenarios," IEEE Wireless Commun. Mag., vol. 20, no. 6, pp. 22-29, Dec. 2013.

[2] H. Bölcskei, "MIMO-OFDM wireless systems: basics, perspectives, and challenges," IEEE Trans. Wireless Commun., vol. 13, no. 4, pp. 31-37, Aug. 2006. 
[3] R. He, Z. Zhong, B. Ai, G. Wang, J. Ding, and A. F. Molisch, "Measurements and analysis of propagation channels in high-speed railway viaducts," IEEE Trans. Wireless Commun., vol. 12, no. 2, pp. 794-805, Feb. 2013.

[4] R. He, Z. Zhong, and B. Ai, "Path loss measurements and analysis for high-speed railway viaduct scene," in Proc. Int. Wireless Commun. and Mobile Computing Conf., Jun. 2010, pp. 266-270.

[5] R. He, Z. Zhong, B. Ai, and J. Ding, "Measurements and analysis of short-term fading behavior for high-speed rail viaduct scenario," in Proc. IEEE Int. Conf. Commun., Jun. 2012, pp. 4563-4567.

[6] R. He, Z. Zhong, B. Ai, J. Ding, Y. Yang, and A. F. Molisch, "Short-term fading behavior in high-speed railway cutting scenario: measurements, analysis, and statistical models," IEEE Trans. Antennas Propag., vol. 61, no. 4, pp. 2209-2222, Apr. 2013.

[7] F. Luan, Y. Zhang, L. Xiao, C. Zhou, and S. Zhou, "Fading characteristics of wireless channel on high-speed railway in hilly terrain scenario," Int. J. Antennas and Propag., vol. 2013, Jan. 2013.

[8] P. Aikio, R. Gruber, and P. Vainikainen, "Wideband radio channel measurements for train tunnels," in Proc. IEEE Veh. Technol. Conf., vol. 1, May 1998, pp. 460-464.

[9] T. Zhou, C. Tao, S. Salous, L. Liu, and Z. Tan, "Channel sounding for high-speed railway communication systems," IEEE Commun. Mag., vol. 53, no. 10, pp. 70-77, Oct. 2015.

[10] B. Ai, X. Cheng, T. Kurner, Z.-D. Zhong, K. Guan, R.-S. He, L. Xiong, D. Matolak, D. Michelson, and C. Briso-Rodriguez, "Challenges toward wireless communications for high-speed railway," IEEE Trans. Intell. Transp. Syst., vol. 15, no. 5, pp. 2143-2158, 2014.

[11] J. Wang, H. Zhu, and N. J. Gomes, "Distributed antenna systems for mobile communications in high speed trains," IEEE J. Sel. Areas Commun., vol. 30, no. 4, pp. 675-683, May 2012.

[12] V. Van Phan, K. Horneman, L. Yu, and J. Vihriälä, "Providing enhanced cellular coverage in public transportation with smart relay systems," in Proc. IEEE Veh. Networking Conf., Dec. 2010, pp. 301-308.

[13] D. T. Fokum and V. S. Frost, "A survey on methods for broadband internet access on trains," IEEE Commun. Surveys Tuts., vol. 12, no. 2, pp. 171-185, Second Quarter 2010.

[14] G. Tingting and S. Bin, "A high-speed railway mobile communication system based on LTE," in Proc. IEEE Int. Conf. Electron. and Inform. Eng., vol. 1, Aug. 2010, pp. 414-417.

[15] B. Ai, K. Guan, M. Rupp, T. Kurner, X. Cheng, X.-F. Yin, Q. Wang, G.Y. Ma, Y. Li, L. Xiong et al., "Future railway services-oriented mobile communications network," IEEE Commun. Mag., vol. 53, no. 10, pp. 78-85, Oct. 2015.

[16] E. Tanghe, W. Joseph, L. Verloock, and L. Martens, "Evaluation of vehicle penetration loss at wireless communication frequencies," IEEE Trans. Veh. Technol., vol. 57, no. 4, pp. 2036-2041, Jul. 2008.

[17] S. Scott, J. Leinonen, P. Pirinen, J. Vihriälä, V. Van Phan, and M. Latvaaho, "A cooperative moving relay node system deployment in a high speed train," in Proc. IEEE Veh. Technol. Conf., Jun. 2013, pp. 1-5.

[18] Y. Sui, J. Vihriälä, A. Papadogiannis, M. Sternad, W. Yang, and T. Svensson, "Moving cells: a promising solution to boost performance for vehicular users," IEEE Commun. Mag., vol. 51, no. 6, pp. 62-68, Jun. 2013.

[19] L. Tian, J. Li, Y. Huang, J. Shi, and J. Zhou, "Seamless dual-link handover scheme in broadband wireless communication systems for high-speed rail," IEEE J. Sel. Areas Commun., vol. 30, no. 4, pp. 708718, May 2012.

[20] W. Luo, R. Zhang, and X. Fang, "A CoMP soft handover scheme for LTE systems in high speed railway," EURASIP J. Wireless Commun. and Networking, vol. 2012, no. 1, pp. 1-9, Jun. 2012.

[21] W. Luo, X. Fang, M. Cheng, and Y. Zhao, "Efficient multiple-group multiple-antenna (MGMA) scheme for high-speed railway viaducts," IEEE Trans. Veh. Technol., vol. 62, no. 6, pp. 2558-2569, Jul. 2013.

[22] X. Cheng and X. Fang, "Principal component analysis based multiplex- ing solution for MIMO systems in high-speed railway," in Proc. High Mobility Wireless Commun. (HMWC), Nov. 2014, pp. 48-52.

[23] J.-Y. Zhang, Z.-H. Tan, and H.-B. Wang, "Optimum capacity of MIMO systems for high-speed railway with spare antenna array," in Proc. IEEE Veh. Technol. Conf., May 2011, pp. 1-4.

[24] N. Sun and J. Wu, "Maximizing spectral efficiency for high mobility systems with imperfect channel state information," IEEE Trans. Wireless Commun., vol. 13, no. 3, pp. 1462-1470, Mar. 2014.

[25] S. Valentin and T. Wild, "Studying the sum capacity of mobile multiuser diversity systems with feedback errors and delay," in Proc. IEEE Veh. Technol. Conf., Sep. 2010, pp. 1-5.

[26] A. Yilmaz and O. Kucur, "Performances of transmit antenna selection, receive antenna selection, and maximal-ratio-combining-based hybrid techniques in the presence of feedback errors," IEEE Trans. Veh. Technol., vol. 63, no. 4, pp. 1976-1982, May 2014.

[27] L. Li, S. A. Vorobyov, and A. B. Gershman, "Transmit antenna selection based strategies in MISO communication systems with low-rate channel state feedback," IEEE Trans. Wireless Commun., vol. 8, no. 4, pp. 16601666, Apr. 2009.

[28] C. Mun, "Transmit-antenna selection for spatial multiplexing with ordered successive interference cancellation," IEEE Trans. Commun., vol. 54, no. 3, pp. 423-429, Mar. 2006

[29] D. A. Gore, R. W. Heath Jr, and A. J. Paulraj, "Transmit selection in spatial multiplexing systems," IEEE Commun. Lett., vol. 6, no. 11, pp. 491-493, Nov. 2002.

[30] R. W. Heath Jr, S. Sandhu, and A. Paulraj, "Antenna selection for spatial multiplexing systems with linear receivers," IEEE Commun. Lett., vol. 5, no. 4, pp. 142-144, Apr. 2001.

[31] R. Klotsche, K. Wünstel, and T.-R. Banniza, "Doppler compensation control for radio transmission,” Jan. 26 2010, US Patent 7,653,347.

[32] J. Li and Y. Zhao, "Radio environment map-based cognitive Doppler spread compensation algorithms for high-speed rail broadband mobile communications," EURASIP Journal Wireless Commun. and Networking, vol. 2012, no. 1, pp. 1-18, Aug. 2012.

[33] "WINNER II channel models, D1.1.2 V1.2 http://http://www.istwinner.org/deliverables.html."

[34] J. Kaleva, A. Tölli, and M. Juntti, "Weighted sum rate maximization for interfering broadcast channel via successive convex approximation," in Proc. IEEE Global Commun. Conf., Dec. 2012, pp. 3838-3843.

[35] Q. Shi, M. Razaviyayn, Z.-Q. Luo, and C. He, "An iteratively weighted MMSE approach to distributed sum-utility maximization for a MIMO interfering broadcast channel," IEEE Trans. Signal Process., vol. 59, no. 9, pp. 4331-4340, Sep. 2011.

[36] D. Tse and P. Viswanath, Fundamentals of Wireless Communication. Cambridge University Press, 2005.

[37] D. J. Love, R. W. Heath, W. Santipach, and M. L. Honig, "What is the value of limited feedback for MIMO channels?" IEEE Commun. Mag., vol. 42, no. 10, pp. 54-59, Oct. 2004.

[38] B. M. Hochwald, T. L. Marzetta, T. J. Richardson, W. Sweldens, and R. Urbanke, "Systematic design of unitary space-time constellations," IEEE Trans. Inf. Theory, vol. 46, no. 6, pp. 1962-1973, Sep. 2000.

[39] A. G. Dabak, C. Lin, E. N. Onggosanusi, and B. Varadarajan, "Codebook and pre-coder selection for closed-loop MIMO," May 24 2011, US Patent 7,949,064.

[40] J. Lee, J.-K. Han, and J. Zhang, "MIMO technologies in 3GPP LTE and LTE-Advanced," EURASIP J. Wireless Commun. and Networking, vol. 2009, Jul. 2009.

[41] J. Roessler, "LTE-Advanced (3GPP Rel. 12) technology introduction white paper," Feb. 2015

[42] A. O. Laiyemo, P. Pirinen, and M. Latva-aho, "Alternative to dynamic rank transmission for LTE mobile relay node system," in Proc. European Wireless Conf., May 2014, pp. 1-6.

[43] W. Weichselberger, M. Herdin, H. Özcelik, and E. Bonek, "A stochastic 
MIMO channel model with joint correlation of both link ends," IEEE Trans. Wireless Commun., vol. 5, no. 1, pp. 90-100, Jan. 2006.

[44] "3rd Generation Partnership Project; Technical Specification Group Radio Access Network; Evolved Universal Terrestrial Radio Access (E-UTRA); Further advancements for E-UTRA physical layer aspects,' 3GPP, Rep. TR 36.814 V9.0.0," Technical Specification, 2010.

[45] J. P. Kermoal, L. Schumacher, K. I. Pedersen, P. E. Mogensen, and F. Frederiksen, "A stochastic MIMO radio channel model with experimental validation," IEEE J. Sel. Areas Commun., vol. 20, no. 6, pp. 1211-1226, Aug. 2002.

[46] "3rd Generation Partnership Project; Technical Specification Group Radio Access Network; Evolved Universal Terrestrial Radio Access (EUTRA); Physical layer procedures," 3GPP, Rep. TR 36.213 V10.6.0," Technical Specification, 2012.

[47] X. He, K. Niu, Z. He, and J. Lin, "Link layer abstraction in MIMOOFDM system," in Proc. IEEE Int. Workshop on Cross Layer Design (IWCLD), Sep. 2007, pp. 41-44.

[48] M. Series, "Guidelines for evaluation of radio interface technologies for IMT-Advanced,” ITU, Tech. Rep., 2009.

[49] S. Tenorio, P. Spence, B. Garriga, J. López, A. García, and M. Arranz, "3G HSPA for broadband communications with high speed vehicles," in Proc. IEEE Veh. Technol. Conf., Apr. 2010, pp. 1-5. 